\title{
Nutrient-sensing nuclear receptors PPAR $\alpha$ and FXR control liver energy balance
}

\author{
Geoffrey A. Preidis, ${ }^{1}$ Kang Ho Kim, ${ }^{2}$ and David D. Moore ${ }^{2}$ \\ ${ }^{1}$ Section of Gastroenterology, Hepatology, and Nutrition, Department of Pediatrics, and ${ }^{2}$ Department of Molecular and Cellular Biology, Baylor College of Medicine, Houston, Texas, USA
}

\begin{abstract}
The nuclear receptors PPAR $\alpha$ (encoded by NR1C1) and farnesoid X receptor (FXR, encoded by NR1H4) are activated in the liver in the fasted and fed state, respectively. PPAR $\alpha$ activation induces fatty acid oxidation, while FXR controls bile acid homeostasis, but both nuclear receptors also regulate numerous other metabolic pathways relevant to liver energy balance. Here we review evidence that they function coordinately to control key nutrient pathways, including fatty acid oxidation and gluconeogenesis in the fasted state and lipogenesis and glycolysis in the fed state. We have also recently reported that these receptors have mutually antagonistic impacts on autophagy, which is induced by PPAR $\alpha$ but suppressed by FXR. Secretion of multiple blood proteins is a major drain on liver energy and nutrient resources, and we present preliminary evidence that the liver secretome may be directly suppressed by PPAR $\alpha$, but induced by FXR. Finally, previous studies demonstrated a striking deficiency in bile acid levels in malnourished mice that is consistent with results in malnourished children. We present evidence that hepatic targets of PPAR $\alpha$ and FXR are dysregulated in chronic undernutrition. We conclude that PPAR $\alpha$ and FXR function coordinately to integrate liver energy balance.
\end{abstract}

\section{Introduction}

The liver is a central mediator of feeding and fasting transitions, pivoting from carbohydrate-based energy accumulation in the fed state to fatty acid oxidation-dependent (FAO-dependent) energy utilization in the fasted state (1). Nuclear receptors and their coregulators are central modulators of these transitions. PPAR $\alpha$ is a well-known inducer of hepatic FAO in the fasted state (2). Although PPAR $\alpha$ is thought to be activated by the high levels of fatty acids present in the fasted liver (3), the exact nature of the endogenous activation signal remains unknown (4).

PPAR $\alpha$ activation also promotes gluconeogenesis (2). Hepatic farnesoid X receptor (FXR) is activated in the fed state by bile acids that return to the liver along with nutrients via the enterohepatic circulation. In addition to maintaining bile acid homeostasis, FXR exerts direct effects on metabolic pathways, including suppression of both gluconeogenesis and lipogenesis (5).

The opposite roles of PPAR $\alpha$ and FXR in nutrient responses, exemplified by their opposite effects on gluconeogenesis, suggest both broad functional interactions and additional counteracting metabolic effects. At the most basic functional level, the two nutrient sensors regulate each other's expression. In the fed state, bile acids activate expression of human PPAR $\alpha$ via direct FXR transactivation, although this is apparently not conserved in mice (6). In the opposite direction, PPAR $\alpha$ activation induces FXR mRNA expression in the fasted mouse liver (7). Thus, each nutrient state primes the other by increasing the expression of the appropriate nutrient sensor.

For additional metabolic effects we have recently shown that these receptors coordinately regulate another fundamen-

Conflict of interest: The authors have declared that no conflict of interest exists. Reference information: J Clin Invest. 2017;127(4):1193-1201.

https://doi.org/10.1172/JCl88893. tal nutrient response in the liver, autophagy (8), as described in more detail below. Recent findings suggest the hepatic secretome is another potential target for complementary control of liver energy balance. Secretion is a very energy-intensive function of the liver, and we recently found that FXR activates the secretion of complement and coagulation factors in human hepatocytes (9). In contrast, earlier studies indicate that PPAR $\alpha$ represses a broader range of secreted proteins in the livers of both mice (10) and cynomolgus monkeys (11). Here we discuss established overlapping and interdependent functional roles of PPAR $\alpha$ and FXR in liver energy balance. We also provide preliminary support for predicted new roles for these nutrient sensors in regulation of the liver secretome and as potential therapeutic targets for chronic malnutrition. The focus on these two receptors should not be taken to exclude important roles for other nuclear receptors $(12,13)$, other nutrient-responsive transcription factors $(14,15)$, or their coregulators $(16,17)$ in central pathways of hepatic energy control. In addition, liver expression of both PPAR $\alpha$ and FXR and many additional key metabolic genes is under independent circadian control, but the complex functional interactions of the circadian clock with nutrient response is beyond the scope of this Review.

\section{PPAR $\alpha$ and FXR as mediators of the fasted} and fed states in the liver

The best-characterized function of PPAR $\alpha$ is to mediate the induction of FAO in the fasted state (2). This is clearly evident from studies of $\mathrm{Nrlc1}^{-/}\left(\mathrm{Ppara}^{-/}\right)$mice, which show dramatic triglyceride accumulation in the fasted state due to loss of the potent PPAR $\alpha$ stimulation of FAO in both peroxisomes and mitochondria (18-20). The idea that PPAR $\alpha$ is activated in the fasted state by elevated levels of free fatty acids is appealing 


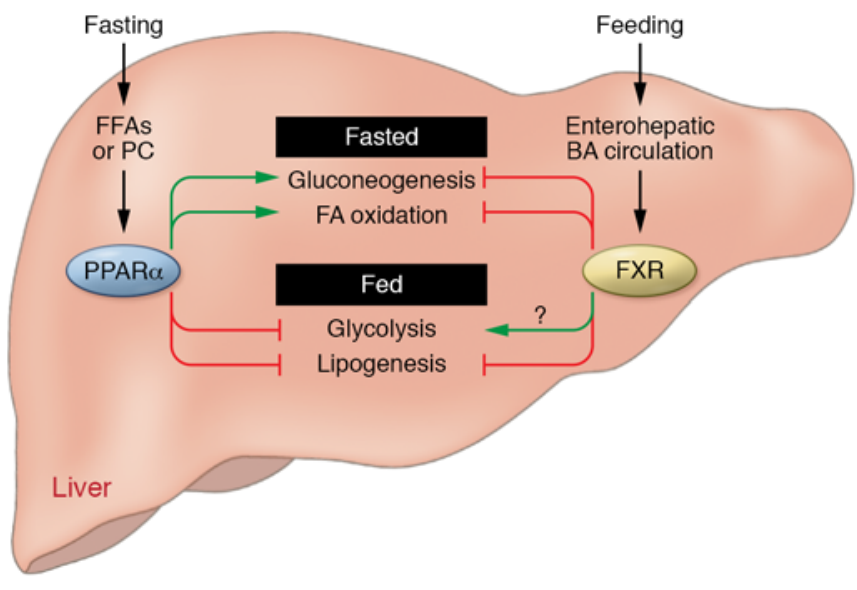

Figure 1. Regulation of fundamental pathways of nutrient metabolism by PPAR $\alpha$ and FXR. The impact of FXR on glycolysis is not well understood. Green arrows indicate activation; red bars indicate repression. FFA, free fatty acid; PC, phosphatidylcholine; BA, bile acid.

because it adds a dual role of the nutrients functioning as signals to induce their own utilization. However, PPAR $\alpha$ activation in the fasted state does not depend on the fatty acids present in the diet (21), and there is evidence that the endogenous ligand is a product of hepatic lipogenesis (4). Direct analysis of lipids bound to liver PPAR $\alpha$ suggested that the endogenous agonist is a relatively abundant phospholipid (22). Endocannabinoids, including oleylethanolamide, have also been identified as potential endogenous agonists (23), and the exact identity of the endogenous PPAR $\alpha$ agonist(s) remains unclear. Interestingly, PPAR $\alpha$ activity is reportedly suppressed in the fed state by a pathway dependent on mTORC1 activation of nuclear receptor co-repressor 1 ; inactivation of $\mathrm{mTORC} 1$ in the fasted state promotes appropriate PPAR $\alpha$ activation (24).

The role of bile acids as endogenous agonist ligands for FXR is well established (25-27). Bile acid homeostasis is an important liver function that controls enterohepatic circulation of bile acids (5). Hepatic FXR is activated by the bile acids that return to the liver accompanied by the nutrients that they help to absorb. A recent report indicates that FXR is stabilized and activated by high glucose levels via O-GlcNAcylation. (28). In addition, FXR activation in the small intestine induces production of FGF-15 (FGF-19 in humans), which exerts insulin-like effects in the liver, including induction of protein and glycogen synthesis (29, 30). FXR activation is a consistent component of the fed state.

\section{Opposing metabolic regulatory functions of PPAR $\alpha$ and FXR in glucose and lipid metabolism}

As noted above, their functions in opposite hepatic nutrient responses suggest that PPAR $\alpha$ and FXR might have opposing effects on central metabolic pathways and liver energy balance. Among such pathways, gluconeogenesis is a key energy homeostasis pathway that has a particularly strong association with the fasting and feeding cycle. We $(31)$ and others $(32,33)$ showed that FXR activation suppresses gluconeogenic genes via induction of the corepressor nuclear receptor SHP. In the opposite direction, hypoglycemia is a key phenotype of the fasted Ppa$\mathrm{ra}^{-/-}$mouse (19), and PPAR $\alpha$ has been identified as a direct activator of a number of gluconeogenic genes $(2,34,35)$. Although there are confounding reports (e.g., ref. 36), gluconeogenesis provides a clear example of complementary but opposing regulatory effects of PPAR $\alpha$ and FXR.

The impact of the two nutrient sensors on pathways of glucose utilization in the fed state has been less studied. There is a report that treatment with the PPAR $\alpha$ agonist fenofibrate decreased expression of glucokinase and pyruvate kinase (PK), which was accompanied by strongly increased expression of the key pyruvate dehydrogenase inhibitor PDK4 and decreased glycolytic flux (37). There are a number of other reports that PPAR $\alpha$ can induce PDK4 in mouse and human hepatocytes $(38,39)$. Suppression of glycolysis by PPAR $\alpha$ is consistent with its function in a fasted state. However, loss of FXR function in $\mathrm{Nr} \mathrm{h} 4^{-1-}$ $\left(\mathrm{Fxr}^{-1}\right)$ mice was associated with increased glycolytic flux in the refed state, indicating that FXR may also suppress glycolysis (40). This suppression was mainly attributed to decreased expression of liver PK (LPK) in the refed Fxr/- livers, but FXR was also found to induce PDK4 expression in rat and human hepatocytes (41).

In lipid metabolism, the clearest impact of either of the two receptors is the activation of FAO by PPAR $\alpha$ in the fasted state (2). Multiple studies with synthetic agonists and Ppara- mice support this conclusion, which is reinforced by the ability of PPAR $\alpha$ agonists to induce the lipolytic metabolic hormone FGF-21 (42). The impact of FXR activation on FAO is less clear, but dietary treatment with the bile acid cholic acid reportedly inhibited primary PPAR $\alpha$ targets, including hepatic mRNA expression of the FAO enzymes acyl-CoA oxidase (Acox1), bifunctional enzyme (Ehhadh), and thiolase (Acaa1a) (43). We found higher expression of FAO genes in $\mathrm{Fxr}^{-1}$ mice (31), and treatment of $d b / d b$ mice with the FXR agonist GW4064 decreased ketogenesis (32). These results indicate that FAO provides another example of opposing effects of PPAR $\alpha$ and FXR. However, FGF-21 has also been identified as a direct target of FXR $(38,44)$, and the ability of FXR activation to induce PDK4 (41) should also support FAO.

We (45) and others (46) have attributed the suppression of lipogenesis upon FXR activation to SHP induction and subsequent suppression of SREBP1c expression. Others have suggested that FXR inhibition of the well-known SREBP1c targets fatty acid synthase (Fasn) and acetyl-CoA carboxylase ( $A c c 1)$ is independent of effects on SREBP1c (40). Inhibition of lipogenesis is consistent with the ability of FXR agonists to improve multiple aspects of the metabolic syndrome, not only in mouse models (31, 32), but also in humans (47); however, it is obviously inconsistent with the induction of this pathway in the fed state. There is less information on the impact of PPAR $\alpha$ on de novo lipogenesis. There is a report that chronic fenofibrate treatment induces lipogenesis in concert with induction of FAO (37), a futile cycle that is not observed in response to fasting, and analysis of Fasn gene expression in fasted and fed Ppara-- mice did not support a lipogenic effect (48). Instead, fenofibrate repressed lipogenic gene expression in high-fat- and high-cholesterol-fed LDL receptor knockout mice (49). Direct assessment of hepatic lipogenesis in hamsters fed a high-fructose diet (50) and, more importantly, 
A

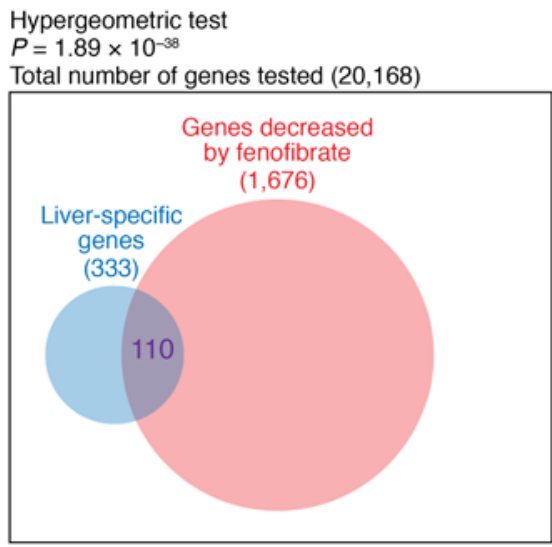

B
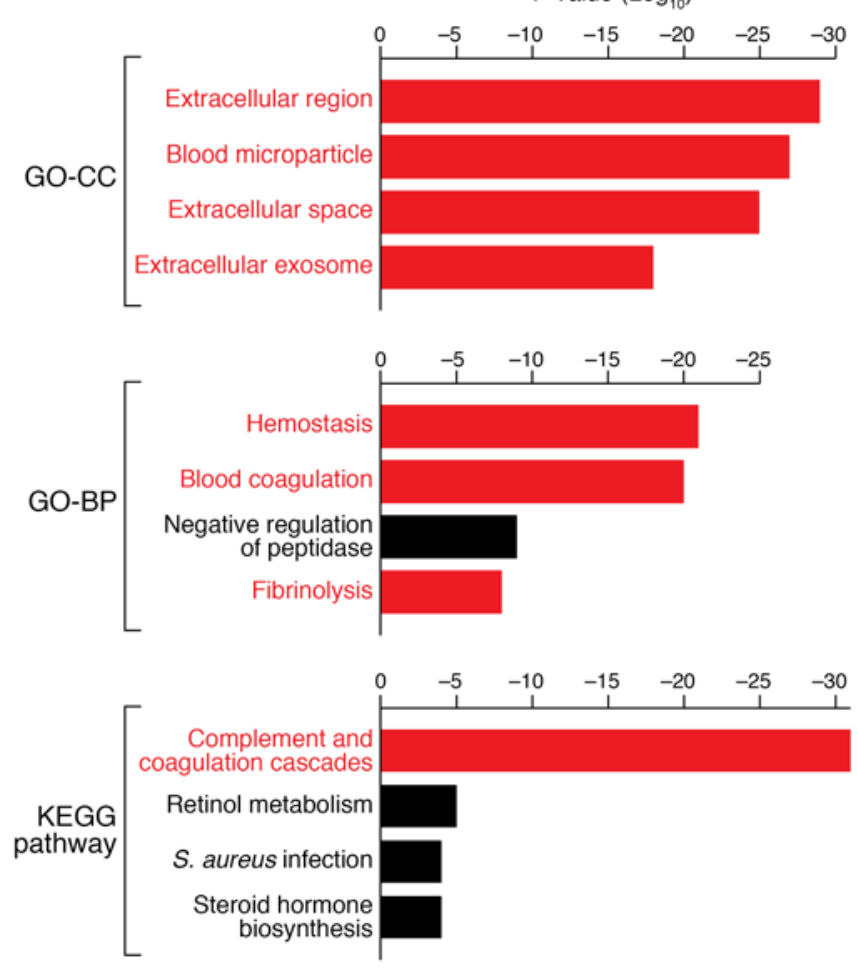

in people with diabetes (51) showed the expected inhibitory effect of fenofibrate on this pathway. The impact of PPAR $\alpha$ and FXR activation on primary pathways of energy metabolism is summarized in Figure 1. These effects are consistent with their expected roles as mediators of the fasted and feeding responses. Their effects are often opposite, particularly their opposing effects on gluconeogenesis and FAO; however, this functional dichotomy is not universal, since both appear to suppress lipogenesis, and they have similar effects on the expression of specific important metabolic regulators, notably PDK4.

\section{PPAR $\alpha$ and FXR coordinately regulate autophagy}

Autophagy is a process by which essential nutrients can be recovered in times of deprivation via recycling of cellular components. The functions of PPAR $\alpha$ and FXR as mediators of the fed and
Figure 2. Suppression of liver-specific gene expression by PPAR $\alpha$ activation. (A) A list of 333 liver-specific genes from Pattern Gene Database (PaGenBase; http://bioinf.xmu.edu.cn/PaGenBase) was compared with an NCBI Gene Expression Omnibus fenofibrate-treated liver microarray (GEO CSE67796). The expression of 145 liver genes was found to be altered $(P<0.01), 110(75.8 \%)$ of which were significantly downregulated by PPAR $\alpha$ agonist treatment (hypergeometric test $P=1.89 \times 10^{-38}$ ). (B) The 110 common genes were subjected to analysis using Database for Annotation, Visualization and Integrated Discovery (DAVID; https://david.ncifcrf.gov/), to address their cellular compartment (gene ontology-cellular compartment [GO-CC]), biological pathways (gene ontology-biological pathways [CO-BP]), and KEGG pathway associations. A majority of the genes encode secreted proteins located in extracellular space.

fasting responses led us to hypothesize that they could have opposing effects on autophagy. We tested this by treating fasted and fed wild-type, $\mathrm{Ppara}^{-/}$, and $\mathrm{Fxr}^{--}$mice with the PPAR $\alpha$ and FXR agonists GW7467 and GW4064. We found that the PPAR $\alpha$ ag onist induced autophagy, even when it was supposed to be off in the fed liver, while the FXR agonist suppressed it, even when it was supposed to be induced in the fasted liver (8). The two agonists had opposing effects on the expression of a large number of autophagy-related genes, and cistromics confirmed that such genes are highly enriched among both receptors' primary targets. We also found that the normal physiologic induction of autophagy in the fasted state was significantly decreased in Ppa$\mathrm{ra}^{-/}$livers. In $\mathrm{Fxr}^{-1}$ livers the expected inhibition of autophagy in the fed state was also blunted. These genetic results strongly reinforce the pharmacologic results and establish physiologic roles for both receptors as mediators of normal nutrient regulation of autophagy. The suppression of autophagy by FXR activation was confirmed by a companion manuscript (52).

Mechanistic studies showed that both PPAR $\alpha$ and FXR could bind to DR-1 motifs (which were previously identified as positive response elements for PPAR $\alpha$ ) in the promoters of autophagy target genes, including genes encoding the autophagosome proteins microtubule-associated protein 1 light chain $3 \alpha$ and - $\beta$ (LC3a and LC3b). FXR was not expected to bind to such sites, but this was confirmed by chromatin immunoprecipitation from wild-type and mutant livers treated with or without the agonists. In accordance with the observed transcriptional repression, FXR binding was associated with GW4064-dependent corepressor recruitment. PPAR $\alpha$ and FXR directly compete for binding to the $L C 3 a$ and $L C 3 b$ promoter sites, with each agonist both increasing the binding of its own receptor and decreasing that of the other.

\section{Regulation of the liver secretome by PPAR $\alpha$ and FXR}

A recent study of the genetic loss of FXR function in humans suggests an unexpected mechanism for PPAR $\alpha$ and FXR to influence hepatic energy balance. We identified four patients from two families with loss-of-function mutations in the FXR (NR1H4) gene (9). Severe neonatal cholestasis was evident in all four patients, and two in one family were successfully treated with liver transplantation, while the other two died prior to one year of age. Cholestasis is expected from the role of FXR in bile acid control but is much more severe in FXR-deficient people than in $\mathrm{Fxr}^{-1}$ mice. All four patients also had coagulopathy. This unex- 
A

Total number of genes tested $(14,026)$

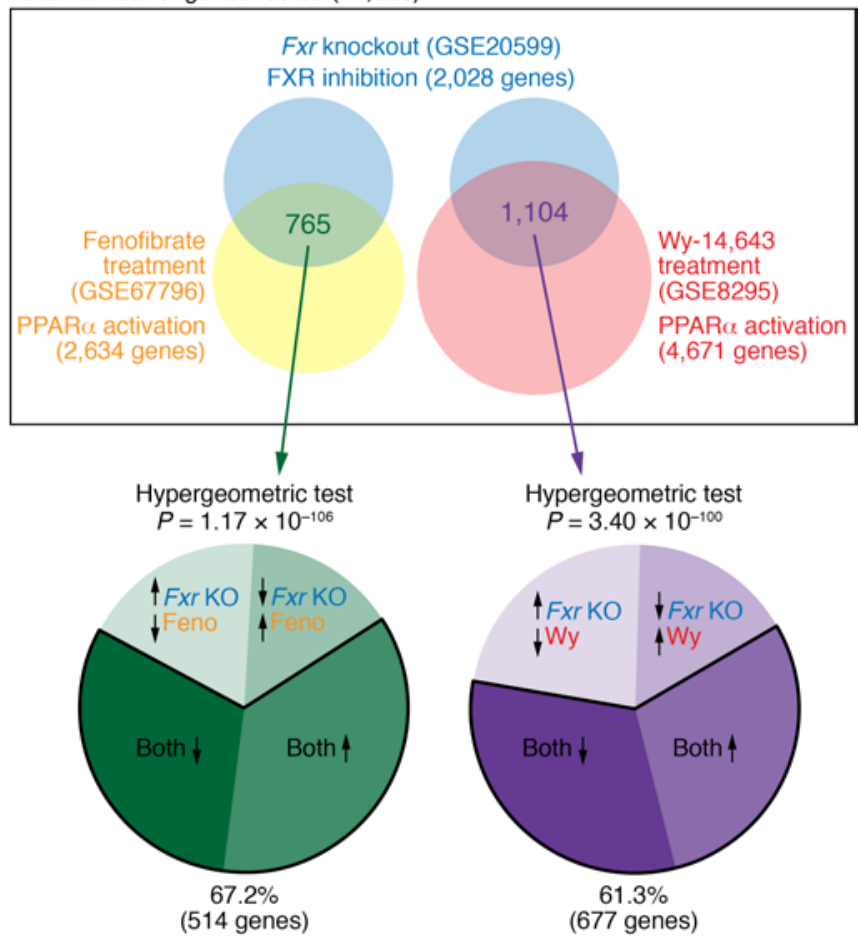

B

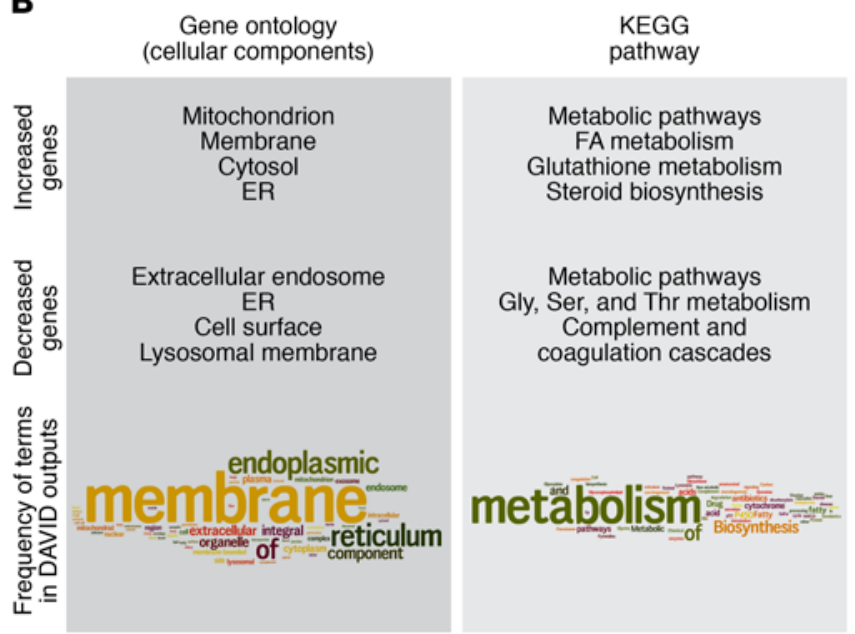

pected phenotype was present from birth, well before the onset of severe liver symptoms, and therefore could not be attributed to end-stage liver disease. Four quite separate lines of evidence support a direct role for FXR as the complement and coagulation pathways. First, we showed that GW4064 induces multiple components of the complement and coagulation pathway in a human hepatocyte cell line (9), extending previous results with fibrinogen. Second, analysis of genome-wide FXR binding studies in mouse and human hepatocytes showed complement and coagulation third on the list of targeted pathways (53). Third, one study associated cholestasis due to diverse etiologies with increased coagulability (54). This was termed "paradoxical" due to the expected association of coagulopathy with severe liver disease but is exactly as expected for a direct transcriptional effect of FXR. Finally, this
Figure 3. Overlapping target genes oppositely regulated by FXR and PPARo. (A) Three microarray data sets (fenofibrate, GSE67796; Fxr knockout, GSE20599; Wy-14,643, GSE8295) were analyzed. Among a total of 14,026 genes, 2,634 (for fenofibrate), 2,028 (for Fxr knockout), and 4,671 genes (for Wy-14,643) were responsive to either PPAR $\alpha$ activation (fenofibrate and $W y-14,643$ treatment) or FXR genetic loss $(P<0.01)$, and many were responsive to both - fenofibrate treatment and Fxr knockout had 765 genes in common, and Fxr knockout and Wy-14,643 treatment had 1,104 genes in common. The majority of overlapping genes were regulated in the same direction (induced or repressed in both; $67.2 \%$ and $61.3 \%$ in each comparison). (B) DAVID analysis (https://david.ncifcrf.gov) of commonly regulated genes in the two comparisons of PPAR $\alpha$ activation/FXR inhibition microarrays. Key aspects of increased or decreased genes were their locations in mitochondrion, membrane, endoplasmic reticulum, or extracellular space and functions as metabolic pathways, complement/coagulation, or fatty acid metabolism. Word cloud analysis (http://www.wordle.net) showed representation of the frequency of terms in the DAVID analysis outputs of the Gene Ontology Cellular Component (left) and KEGG pathway (right) $(P<0.05)$.

induction result was supported by a modest but significant increase in clotting rate in people with non-alcoholic steatohepatitis treated with obeticholic acid, a semisynthetic FXR agonist (47). Overall, it is clear that FXR directly regulates components of the complement and coagulation cascade.

There is extensive evidence that PPAR $\alpha$ represses the same complement and coagulation pathways described above. Gene array profiling of the effects of the PPAR $\alpha$ agonist ciprofibrate in the cynomolgus monkey showed the expected upregulation of FAO and other known targets but also strong downregulation of many complement and coagulation genes (11). In mice the complement and coagulation cascades were repressed by fasting (55) and were among the strongest negative targets of fenofibrate (10); they were also repressed by PPAR $\alpha$ agonists in rats (56). Repression of fibrinogen gene expression by fenofibrate was lost in $\mathrm{Ppara}^{-/-}$mice (57). Complement genes are also repressed by fibrates in human hepatocytes (58), and fibrate treatments decrease fibrinogen levels in human serum $(59,60)$. The combination of either gemfibrozil (52) or fenofibrate (53) with warfarin markedly decreases clotting rates, although this has been attributed to pharmacodynamic effects related to displacement of warfarin from plasma albumin (61).

The metabolic functions of FXR and PPAR $\alpha$ seem quite unrelated to coagulation; however, we have identified a potential linkage based on the idea that secretion is, in a sense, the inverse of autophagy: amino acids and energy in proteins are recovered by autophagy but are lost by secretion. Secretion is directly relevant to energy balance because it is arguably the most resource- and energy-intensive process in the liver. Since approximately $40 \%$ of all hepatocyte mRNAs encode secretory proteins (62), nearly half of the total protein produced by each hepatocyte is simply lost via secretion. This is a huge drain on resources. For the human liver, the daily total protein secretion is approximately 25 grams per day $(63,64)$, which corresponds to roughly half of the recommended daily protein consumption. Decreases in oxygen consumption upon cycloheximide treatment indicate that up to $10 \%$ of hepatocyte ATP goes to production of these secreted proteins (65). Thus, we extend the complement and coagulation pathways to the entire hepatocyte secretome and suggest that FXR licenses the process of secretion in the nutrient-rich fed state while PPAR $\alpha$ spares resources by repressing it in the fasted state. 
The regulation of the process of secretion in the fed and fasted states has been known for decades. Studies nearly 40 years ago showed that essentially all albumin mRNA is present on secretory rough endoplasmic reticulum polysomes in the fed rat liver, as expected, but the majority of these transcripts are released into the untranslated post-ribosomal pool in the fasted liver (66). In accord with this, human albumin production shows a two-fold daily fluctuation in production in the fed and fasted states (63), although this does not acutely affect serum albumin levels due to the approximately two-week half-life of the protein (64). Thus, the proposed PPAR $\alpha$ and FXR effects represent the addition of a new transcriptional mechanism to a well-established regulatory effect. An intriguing aspect of this hypothesis is that it is completely independent of the diverse functions of the secreted proteins, which presumably are regulated by additional inputs. Instead we suggest that the impact of the secretome on hepatocyte energy balance is the basis for its apparent regulation by PPAR $\alpha$ and FXR.

Available genome-wide profiling studies provide some support for the prediction that PPAR $\alpha$ and FXR coordinately control the hepatocyte secretome. In one profile of fenofibrate effects in mice (67), nearly half (145/333) of a panel of liver-specific genes were altered by PPAR $\alpha$ agonist treatment, and of these 110 (75.8\%) were repressed (Figure 2A). As confirmed with analyses using Gene Ontology (www.geneontology.org) and Kyoto Encyclopedia of Genes and Genomes (KEGG; www.kegg.jp/) (Figure 2B), these suppressed genes are highly enriched for secreted proteins, including many in addition to the components of the complement and coagulation cascades. This enrichment of PPAR $\alpha$-repressed secretory targets is highly statistically significant.

A further analysis compared transcripts affected by genetic loss of FXR with those altered by the PPAR $\alpha$ agonists fenofibrate or Wy-14,643. The two comparisons identified more than 750 and 1,100 transcripts affected by both loss of FXR function and gain of PPAR $\alpha$ function. In accordance with the opposite nutrient roles of the two nuclear receptors, concordant responses - either increased or decreased in both PPAR $\alpha$ agonist and Fxr knockout - were much more common (67\%) than discordant responses in the fenofibrate comparison (Figure 3A). Similarly, in the Wy-14,643 comparison, $61 \%$ of the common genes were altered in the same direction by PPAR $\alpha$ activation and FXR inactivation. The increase in coordinate responses relative to discordant responses was highly statistically significant with the hypergeometric test. As expected, pathway analysis of the gene set representing PPAR $\alpha$ activation and FXR inactivation highlighted FAO and additional PPAR $\alpha$ targets relevant to energy balance, including mitochondrial function (Figure 3B). Additionally, secretome components were highly enriched in the gene set repressed by both PPAR $\alpha$ agonists and Fxr knockout, as determined using both Gene Ontology and KEGG pathway analysis (Figure 3B). In the Gene Ontology analysis, nearly a quarter of the 412 genes in this category appeared in extracellular exosome (GO:0070062; 105 of $412 ; P=8.92 \times 10^{-12}$ ), extracellular region (GO:9995576; 134 of $412 ; P=2.16 \times 10^{-7}$ ) or extracellular vesicle (GO:1903461; 105 of $\left.412 ; P=1.31 \times 10^{-11}\right)$. Overall these results strongly support the predominance of the opposite effects of PPAR $\alpha$ and FXR agonists but also leave open the possibilities of convergent effects, such as those observed with lipogenesis.

\section{Altered nuclear receptor signaling in extreme nutrient deprivation}

A more complete understanding of coordinate regulation of nutrient-sensing nuclear receptors has a potential impact on numerous pathologic conditions affected by liver energy imbalance. Although the current research focus is largely on overnutritionassociated metabolic syndrome, there is also great potential for relevance in child undernutrition. Globally, undernutrition contributes to half of all deaths of children under five years of age (68). When present in early childhood, undernutrition confers longterm deficits in growth potential and IQ, along with increased risk of obesity and related disorders later in life (69). Despite recent progress by the Millennium Development Goals (70), more than 113 million children worldwide remain underweight (71).

Similar to the acutely fasted state, chronic undernutrition requires the host to conserve and recycle amino acids and energy. Accordingly, signs of suppressed secretion and enhanced autophagy have been identified in protein calorie-restricted humans. Most notably, hypoalbuminemia is a hallmark of the severe, edematous form of undernutrition known as kwashiorkor (72). Likewise, dozens of studies have reported low plasma levels of complement proteins, in particular C3, in undernourished children (73). Suppressed secretion of complement likely contributes to the impaired cell-mediated immunity that is a feature of many forms of undernutrition (74).

Two other important secretory defects have been observed in chronic undernutrition. First, undernourished children have decreased plasma concentrations of coagulation factors with elevated prothrombin time (75-83). This coagulopathy often manifests as bruising but in rare cases can result in severe gastrointestinal hemorrhage $(75,81)$. Although the vitamin $\mathrm{K}$-dependent factors II, VII, IX, and $\mathrm{X}$ are often decreased, many cases of coagulopathy in severe undernutrition are associated with decreased vitamin $\mathrm{K}$-independent coagulation factors $(80,82)$ $(71,73)$ or are refractory to intravenous vitamin $\mathrm{K}$ therapy $(75)$. More recently, coagulopathy was reported in a cohort of young adults with severe anorexia nervosa (84). Second, undernourished children have decreased concentrations of small intestinal conjugated bile acids (85-87), which leads to impaired lipid solubilization (88). Decreased bile acid secretion likely contributes to the observed dietary fat malabsorption (89-95), fat-soluble vitamin deficiencies (96), and small intestinal bacterial overgrowth (97102). Decreased bile acids might also help explain why therapeutic refeeding often fails to result in sustained growth (103-105). Invasive small bowel sampling is no longer performed strictly for research purposes; however, a more recent study investigated fecal bile acids obtained from Malawian children with severe acute undernutrition who were admitted for inpatient nutritional rehabilitation. Nutritional recovery was associated with a nearly seven-fold increase in median concentration of conjugated bile acids in stool, compared with levels measured at hospital admission (106). Taken together, these findings suggest that undernourished individuals have decreased secretion of albumin, complement proteins, coagulation factors, and bile acids.

In addition to reduced secretion, there is also evidence that chronic protein calorie undernutrition upregulates autophagy. Numerous autophagosomes were identified with electron micros- 
copy analysis of liver biopsy samples obtained from young adults with severe anorexia nervosa (84). Another recent study examined autophagic gene transcript and protein expression levels in skeletal muscle biopsies from otherwise healthy adults who had maintained a calorie-restricted diet for three or more years. Compared with sedentary individuals consuming Western diets, skeletal muscle from those with long-term calorie restriction contained numerous upregulated autophagic markers (107).

Overall, the decreased secretion and increased autophagy suggests that chronic protein calorie undernutrition in some respects parallels the acutely fasted state, with an imbalance between PPAR $\alpha$ and FXR signaling. Fatty acids mobilized from peripheral fat stores might serve as endogenous PPAR $\alpha$ ligands, whereas lack of FXR signaling might result from decreased bile acid synthesis. Indeed, we (108) and others (109) have reported striking decreases in conjugated bile acids in mouse models of protein energy undernutrition. Our metabolomic screen also revealed evidence of increased FAO and autophagy (108). However, recent studies using a rat model of undernutrition induced by a low-protein diet revealed hypoalbuminemia, loss of peroxisomes in hepatocytes, and impaired peroxisomal and mitochondrial function - effects that were partially ameliorated by the PPAR $\alpha$ agonist fenofibrate (110). These data suggest a lack of PPAR $\alpha$ signaling in this proteindeficient rat model, raising the possibility that endogenous ligands fail to activate PPAR $\alpha$ and the resulting homeostatic effects. Alternatively, pathways other than PPAR $\alpha$ signaling might contribute to FAO and energy-sparing suppression of secretion during chronic macronutrient deprivation.

Our quest to better understand nuclear receptor function and regulation in the undernourished host faces several challenges. These include integrating the coordinate activities of other nuclear receptors (such as vitamin D receptor) in settings of highly prevalent micronutrient deficiencies; evaluating liver secretory effects in the context of occult or overt liver diseases, including those related to undernutrition-associated steatosis or infections with helminth or viral pathogens; and navigating the challenges inherent to performing translational studies with extremely vulnerable patient populations. Nonetheless, we anticipate that defective PPAR $\alpha$ or FXR signaling may be relevant to vexing questions, such as why some children are susceptible to hypoalbuminemia and kwashiorkor, whereas others develop marasmus, the non-edematous form of undernutrition. Proper nuclear receptor activation may also provide an opportunity to improve refeeding practices, reduce mortality, enhance catch-up growth, and decrease long-term metabolic complications of early-life undernutrition.

\section{Conclusions}

Gemfibrozil and fenofibrate are the two fibrates currently approved for use in the US. Based on their ability to lower serum triglycerides and decrease cardiovascular events, their usage approximately doubled in the US from 2004 to 2009 (111). In 2010, results from the Action to Control Cardiovascular Risk in Diabetes (ACCORD) trial confirmed that the addition of fenofibrate to simvastatin decreased cardiovascular risk in the subset of simvastatin-treated patients with relatively high triglycerides and low HDL (112), and it is likely that fibrate usage will continue to increase. The FXR Ligand Obeticholic Acid for Non-cirrhotic, Non-alcoholic Steatohepatitis (FLINT) trial recently showed that the bile acid derivative obeticholic acid improves multiple parameters of metabolic syndrome (47). Thus, obeticholic acid, which was very recently approved for the treatment of cholestatic liver disease (113), may also come into common use for treating metabolic syndrome.

The common beneficial impact of both PPAR $\alpha$ and FXR agonists on aspects of metabolic syndrome seems inconsistent with many of the results outlined above. How can activation of two receptors with apparently opposite metabolic functions have similar beneficial effects? One possibility relates to the ability of both to suppress lipogenesis. As suggested by the late Denis McGarry, increased lipogenesis in the insulin-resistant liver can drive a vicious cycle in which increased steatosis reinforces insulin resistance, which in turn drives even more steatosis (114). In response to activation of several nuclear receptors, including FXR and possibly PPAR $\alpha$, this cycle may be reversed (115). In this model, inhibition of lipogenesis in response to receptor activation improves fatty liver and promotes insulin sensitivity, which in turn further suppresses lipogenesis and propagates as a beneficial cycle. For PPAR $\alpha$, this model is consistent with results in mice (116) and with some reports in humans that suggest insulin sensitivity is improved in response to fenofibrate treatment (117-120). However, the model is not supported by other clinical studies that have not shown either decreased steatosis (121) or increased insulin sensitivity in humans in response to fenofibrate (121-124).

There are still hundreds of genes that respond in the same direction to activation of the two nuclear receptors that may provide additional specific mechanisms. More broadly, we conclude that the preponderance of opposing effects of PPAR $\alpha$ and FXR (Figure 3) correlates with their complementary activation in the fed and fasted states. This means that the two receptors do not simply cancel each other's effects. Instead, in specific pathways such as activation and suppression of gluconeogenesis and autophagy in the fasted and fed states, the two nutrient sensors function coordinately in the appropriate nutrient contexts to appropriately regulate metabolic flux and energy balance. This suggests that specific activation of each in the correct portion of the daily cycle could promote proper metabolic balance via quite distinct mechanisms. Perhaps combinations of PPAR $\alpha$ and FXR agonists would have reinforcing or synergistic beneficial effects if it were pharmacologically possible for each to target only the appropriate state.

\section{Acknowledgments}

This work was supported by NIDDK grant U24 DK097748. DDM is supported by the Robert R.P. Doherty Jr. Welch Chair in Science (grant Q-0022). GAP is supported by NIH training grant T32DK007664; by the US Public Health Service grant P30DK56338, which funds the Texas Medical Center Digestive Disease Center; and by the North American Society for Pediatric Gastroenterology, Hepatology and Nutrition Foundation/Nestle Nutrition Research Young Investigator Development Award.

Address correspondence to: David D. Moore, Department of Molecular and Cellular Biology, 1 Baylor Plaza, Houston, Texas 77030, USA. Phone: 713.798.3313; E-mail: moore@bcm.edu. 
1. Rui L. Energy metabolism in the liver. Compr Physiol. 2014;4(1):177-197.

2. Kersten S. Integrated physiology and systems biology of PPAR $\alpha$. Mol Metab. 2014;3(4):354-371.

3. Pawlak M, Lefebvre P, Staels B. Molecular mechanism of PPAR $\alpha$ action and its impact on lipid metabolism, inflammation and fibrosis in non-alcoholic fatty liver disease. J Hepatol. 2015;62(3):720-733.

4. Lodhi IJ, Wei X, Semenkovich CF. Lipoexpediency: de novo lipogenesis as a metabolic signal transmitter. Trends Endocrinol Metab. 2011;22(1):1-8.

5. Matsubara T, Li F, Gonzalez FJ. FXR signaling in the enterohepatic system. Mol Cell Endocrinol. 2013;368(1-2):17-29.

6. Pineda Torra I, Claudel T, Duval C, Kosykh V, Fruchart JC, Staels B. Bile acids induce the expression of the human peroxisome proliferator-activated receptor $\alpha$ gene via activation of the farnesoid X receptor. Mol Endocrinol. 2003;17(2):259-272.

7. Kok $\mathrm{T}$, et al. Induction of hepatic $\mathrm{ABC}$ transporter expression is part of the PPAR $\alpha$-mediated fasting response in the mouse. Gastroenterology. 2003;124(1):160-171.

8. Lee JM, et al. Nutrient-sensing nuclear receptors coordinate autophagy. Nature. 2014;516(7529):112-115.

9. Gomez-Ospina N, et al. Mutations in the nuclear bile acid receptor FXR cause progressive familial intrahepatic cholestasis. Nat Commun. 2016;7:10713.

10. Lu Y, Boekschoten MV, Wopereis S, Müller M, Kersten S. Comparative transcriptomic and metabolomic analysis of fenofibrate and fish oil treatments in mice. Physiol Genomics. 2011;43(23):1307-1318.

11. Cariello NF, et al. Gene expression profiling of the PPAR-alpha agonist ciprofibrate in the cynomolgus monkey liver. Toxicol Sci. 2005;88(1):250-264.

12. Giguère V. Transcriptional control of energy homeostasis by the estrogen-related receptors. Endocr Rev. 2008;29(6):677-696.

13. Gerhart-Hines Z, Lazar MA. Rev-erb $\alpha$ and the circadian transcriptional regulation of metabolism. Diabetes Obes Metab. 2015;17 Suppl 1:12-16.

14. Altarejos JY, Montminy M. CREB and the CRTC co-activators: sensors for hormonal and metabolic signals. Nat Rev Mol Cell Biol. 2011;12(3):141-151.

15. Pajvani UB, Accili D. The new biology of diabetes. Diabetologia. 2015;58(11):2459-2468.

16. Li S, Lin JD. Transcriptional control of circadian metabolic rhythms in the liver. Diabetes Obes Metab. 2015;17(suppl 1):33-38.

17. Stashi E, York B, O'Malley BW. Steroid receptor coactivators: servants and masters for control of systems metabolism. Trends Endocrinol Metab. 2014;25(7):337-347.

18. Lee SS, et al. Targeted disruption of the alpha isoform of the peroxisome proliferator-activated receptor gene in mice results in abolishment of the pleiotropic effects of peroxisome proliferators. Mol Cell Biol. 1995;15(6):3012-3022.

19. Kersten S, Seydoux J, Peters JM, Gonzalez FJ, Desvergne B, Wahli W. Peroxisome proliferator-activated receptor $\alpha$ mediates the adaptive response to fasting. J Clin Invest. 1999;103(11):1489-1498.

20. Leone TC, Weinheimer CJ, Kelly DP. A critical role for the peroxisome proliferator-activated receptor alpha (PPAR $\alpha$ ) in the cellular fasting response: the PPAR $\alpha$-null mouse as a model of fatty acid oxidation disorders. Proc Natl Acad Sci U S A. 1999;96(13):7473-7478.

21. Polizzi A, et al. Hepatic fasting-induced PPAR $\alpha$ activity does not depend on essential fatty acids. Int J Mol Sci. 2016;17(10):E1624.

22. Chakravarthy MV, et al. Identification of a physiologically relevant endogenous ligand for PPARo in liver. Cell. 2009;138(3):476-488.

23. Fu J, et al. Oleylethanolamide regulates feeding and body weight through activation of the nuclear receptor PPAR- $\alpha$. Nature. 2003;425(6953):90-93.

24. Sengupta S, Peterson TR, Laplante M, Oh S, Sabatini DM. mTORC1 controls fasting-induced ketogenesis and its modulation by ageing. Nature. 2010;468(7327):1100-1104.

25. Parks DJ, et al. Bile acids: natural ligands for an orphan nuclear receptor. Science. 1999;284(5418):1365-1368.

26. Makishima M, et al. Identification of a nuclear receptor for bile acids. Science. 1999;284(5418):1362-1365.

27. Wang H, Chen J, Hollister K, Sowers LC, Forman BM. Endogenous bile acids are ligands for the nuclear receptor FXR/BAR. Mol Cell. 1999;3(5):543-553.

28. Berrabah W, et al. Glucose sensing O-GlcNAcylation pathway regulates the nuclear bile acid receptor farnesoid X receptor (FXR). Hepatology. 2014;59(5):2022-2033.

29. Kir S, et al. FGF19 as a postprandial, insulin-independent activator of hepatic protein and glycogen synthesis. Science. 2011;331(6024):1621-1624.

30. Kliewer SA, Mangelsdorf DJ. Bile acids as hormones: the FXR-FGF15/19 pathway. Dig Dis. 2015;33(3):327-331.

31. Ma K, Saha PK, Chan L, Moore DD. Farnesoid X receptor is essential for normal glucose homeostasis. J Clin Invest. 2006;116(4):1102-1109.

32. Zhang Y, et al. Activation of the nuclear receptor FXR improves hyperglycemia and hyperlipidemia in diabetic mice. Proc Natl Acad Sci U S A. 2006;103(4):1006-1011.

33. Cariou B, et al. The farnesoid X receptor modulates adiposity and peripheral insulin sensitivity in mice. J Biol Chem. 2006;281(16):11039-11049.

34. Jitrapakdee S, et al. The peroxisome proliferatoractivated receptor-gamma regulates murine pyruvate carboxylase gene expression in vivo and in vitro. J Biol Chem. 2005;280(29):27466-27476.

35. Patsouris D, et al. PPAR $\alpha$ governs glycerol metabolism. J Clin Invest. 2004;114(1):94-103.

36. Stayrook KR, et al. Regulation of carbohydrate metabolism by the farnesoid X receptor. Endocrinology. 2005;146(3):984-991.

37. Oosterveer MH, et al. Fenofibrate simultaneously induces hepatic fatty acid oxidation, synthesis, and elongation in mice. J Biol Chem. 2009;284(49):34036-34044.

38. Motojima K, Seto K. Fibrates and statins rapidly and synergistically induce pyruvate dehydrogenase kinase 4 mRNA in the liver and muscles of mice. Biol Pharm Bull. 2003;26(7):954-958.
39. Janssen AW, et al. The impact of PPAR $\alpha$ activation on whole genome gene expression in human precision cut liver slices. BMC Genomics. 2015;16:760.

40. Duran-Sandoval D, et al. The farnesoid X receptor modulates hepatic carbohydrate metabolism during the fasting-refeeding transition. J Biol Chem. 2005;280(33):29971-29979.

41. Savkur RS, Bramlett KS, Michael LF, Burris TP. Regulation of pyruvate dehydrogenase kinase expression by the farnesoid X receptor. Biochem Biophys Res Commun. 2005;329(1):391-396.

42. Inagaki $\mathrm{T}$, et al. Endocrine regulation of the fasting response by PPAR $\alpha$-mediated induction of fibroblast growth factor 21. Cell Metab. 2007;5(6):415-425.

43. Sinal CJ, Yoon M, Gonzalez FJ. Antagonism of the actions of peroxisome proliferatoractivated receptor- $\alpha$ by bile acids. J Biol Chem. 2001;276(50):47154-47162.

44. Cyphert HA, Ge X, Kohan AB, Salati LM, Zhang Y, Hillgartner FB. Activation of the farnesoid X receptor induces hepatic expression and secretion of fibroblast growth factor 21. J Biol Chem. 2012;287(30):25123-25138.

45. Watanabe $\mathrm{M}$, et al. Bile acids lower triglyceride levels via a pathway involving FXR, SHP, and SREBP-1c. J Clin Invest. 2004;113(10):1408-1418.

46. Yamagata $\mathrm{K}$, et al. Bile acids regulate gluconeogenic gene expression via small heterodimer partner-mediated repression of hepatocyte nuclear factor 4 and Foxo1. J Biol Chem. 2004;279(22):23158-23165.

47. Neuschwander-Tetri BA, et al. Farnesoid X nuclear receptor ligand obeticholic acid for noncirrhotic, non-alcoholic steatohepatitis (FLINT): a multicentre, randomised, placebo-controlled trial. Lancet. 2015;385(9972):956-965.

48. Montagner A, et al. Liver PPAR $\alpha$ is crucial for whole-body fatty acid homeostasis and is protective against NAFLD. Gut. 2016;65(7):1202-1214

49. Srivastava RA, Jahagirdar R, Azhar S, Sharma S, Bisgaier CL. Peroxisome proliferator-activated receptor- $\alpha$ selective ligand reduces adiposity, improves insulin sensitivity and inhibits atherosclerosis in LDL receptor-deficient mice. Mol Cell Biochem. 2006;285(1-2):35-50.

50. Rizvi F, et al. Antidyslipidemic action of fenofibrate in dyslipidemic-diabetic hamster model. Biochem Biophys Res Commun. 2003;305(2):215-222.

51. Forcheron F, Cachefo A, Thevenon S, Pinteur C, Beylot M. Mechanisms of the triglyceride- and cholesterol-lowering effect of fenofibrate in hyperlipidemic type 2 diabetic patients. Diabetes. 2002;51(12):3486-3491.

52. Seok $\mathrm{S}$, et al. Transcriptional regulation of autophagy by an FXR-CREB axis. Nature. 2014;516(7529):108-111.

53. Zhan L, et al. Genome-wide binding and transcriptome analysis of human farnesoid $\mathrm{X}$ receptor in primary human hepatocytes. PLoS One. 2014;9(9):e105930.

54. Magnusson M, Fischler B, Svensson J, Petrini P, Schulman S, Németh A. Bile acids and coagulation factors: paradoxical association in children with chronic liver disease. Eur J Gastroenterol Hepatol. 2013;25(2):152-158.

55. Hakvoort TB, et al. Interorgan coordination of 
the murine adaptive response to fasting. J Biol Chem. 2011;286(18):16332-16343.

56. Tamura K, Ono A, Miyagishima T, Nagao T, Urushidani T. Profiling of gene expression in rat liver and rat primary cultured hepatocytes treated with peroxisome proliferators. J Toxicol Sci. 2006;31(5):471-490.

57. Kockx M, et al. Fibrates suppress fibrinogen gene expression in rodents via activation of the peroxisome proliferator-activated receptor-alpha. Blood. 1999;93(9):2991-2998.

58. Rakhshandehroo M, Hooiveld G, Müller M, Kersten S. Comparative analysis of gene regulation by the transcription factor PPAR $\alpha$ between mouse and human. PLoS One. 2009;4(8):e6796.

59. Watts GF, Dimmitt SB. Fibrates, dyslipoproteinaemia and cardiovascular disease. Curr Opin Lipidol. 1999;10(6):561-574.

60. Sahebkar A, et al. Head-to-head comparison of statins versus fibrates in reducing plasma fibrinogen concentrations: A systematic review and meta-analysis. Pharmacol Res. 2016;103:236-252.

61. Lilja JJ, Backman JT, Neuvonen PJ. Effect of gemfibrozil on the pharmacokinetics and pharmacodynamics of racemic warfarin in healthy subjects. Br J Clin Pharmacol. 2005;59(4):433-439.

62. Uhlen M, et al. Proteomics. Tissue-based map of the human proteome. Science. 2015;347(6220):1260419.

63. De Feo P, Horber FF, Haymond MW. Meal stimulation of albumin synthesis: a significant contributor to whole body protein synthesis in humans. Am J Physiol. 1992;263(4 pt 1):E794-E799.

64. Margarson MP, Soni N. Serum albumin: touchstone or totem? Anaesthesia. 1998;53(8):789-803.

65. Kelly JM, McBride BW. The sodium pump and other mechanisms of thermogenesis in selected tissues. Proc Nutr Soc. 1990;49(2):185-202.

66. Yap SH, Strair RK, Shafritz DA. Effect of a short term fast on the distribution of cytoplasmic albumin messenger ribonucleic acid in rat liver. Evidence for formation of free albumin messenger ribonucleoprotein particles. J Biol Chem. 1978;253(14):4944-4950.

67. Ferguson LB, Most D, Blednov YA, Harris RA. PPAR agonists regulate brain gene expression: relationship to their effects on ethanol consumption. Neuropharmacology. 2014;86:397-407.

68. Black RE, et al. Maternal and child undernutrition and overweight in low-income and middle-income countries. Lancet. 2013;382(9890):427-451.

69. Guerrant RL, DeBoer MD, Moore SR, Scharf RJ, Lima AA. The impoverished gut - a triple burden of diarrhoea, stunting and chronic disease. Nat Rev Gastroenterol Hepatol. 2013;10(4):220-229.

70. Brown NJ, Platt MP, Beattie RM. Women, children, and global public health: beyond the millennium development goals. BMJ. 2015;350:h1755.

71. de Onis M, Blössner M, Borghi E, Frongillo EA, Morris R. Estimates of global prevalence of childhood underweight in 1990 and 2015. JAMA. 2004;291(21):2600-2606

72. Coulthard MG. Oedema in kwashiorkor is caused by hypoalbuminaemia. Paediatr Int Child Health. 2015;35(2):83-89.

73. Rytter MJ, Kolte L, Briend A, Friis H, Christensen VB. The immune system in children with malnutrition - a systematic review. PLoS One. 2014;9(8):e105017.

74. Smythe PM, et al. Thymolymphatic deficiency and depression of cell-mediated immunity in protein-calorie malnutrition. Lancet. 1971;2(7731):939-943.

75. Rapoport S, Dodd K. Hypoprothrombinemia in infants with diarrhea. Am J Dis Child. 1946;71:611-617.

76. Matoth Y. Plasma prothrombin in infantile diarrhea. AMA Am J Dis Child. 1950;80(6):944-954.

77. Chavez R, Frenk S, Galvan RR, Gomez F, Munoz JC, Vazquez J. Mortality in second and third degree malnutrition. J Trop Pediatr (Lond). 1956;2(2):77-83.

78. Merskey C, Hansen JD. Blood coagulation defects in kwashiorkor and infantile gastroenteritis. $\mathrm{Br} \mathrm{J}$ Haematol. 1957;3(1):39-49.

79. Kahn E, Stein H. Purpura in kwashiorkor. Br Med J. 1959;2(5141):66-68.

80. Hassanein EA, Tankovsky I. Disturbances of coagulation mechanism in protein-calorie malnutrition. Trop Geogr Med. 1973;25(2):158-162.

81. Hassanein EA, Tankovsky I. Disseminated intravascular clotting in kwashiorkor. Arch Dis Child. 1975;50(4):308-310.

82. Jain K, Singh SD, Mukerjee DP. Observations on thrombolastographic patterns and coagulation changes in malnutrition (Marasmus and Kwashiorkor). Indian Pediatr. 1979;16(12):1115-1119.

83. Akinyinka OO, Falade AG, Ogbechie CO. Prothrombin time as an index of mortality in kwashiorkor. Ann Trop Paediatr. 1990;10(1):85-88.

84. Rautou PE, et al. Acute liver cell damage in patients with anorexia nervosa: a possible role of starvation-induced hepatocyte autophagy. Gastroenterology. 2008;135(3):840-848, 848.e1.

85. Schneider RE, Viteri FE. Luminal events of lipid absorption in protein-calorie malnourished children; relationship with nutritional recovery and diarrhea. II. Alterations in bile acid content of duodenal aspirates. Am J Clin Nutr. 1974;27(8):788-796.

86. Mehta HC, Saini AS, Singh H, Dhatt PS. Biochemical aspects of malabsorption in marasmus. $\mathrm{Br} \mathrm{J}$ Nutr. 1984;51(1):1-6.

87. Mehta HC, Saini AS, Singh H, Dhatt PS. Biochemical aspects of malabsorption in marasmus: effect of dietary rehabilitation. Br J Nutr. 1985;54(3):567-575.

88. Schneider RE, Viteri FE. Luminal events of lipid absorption in protein-calorie malnourished children; relationship with nutritional recovery and diarrhea. I. Capacity of the duodenal content to achieve micellar solubilization of lipids. Am JClin Nutr. 1974;27(8):777-787.

89. Dean RF. Treatment and prevention of kwashiorkor. Bull World Health Organ. 1953;9 (6):767-783.

90. Trowell HC. Clinical aspects of the treatment of kwashiorkor. Ann N Y Acad Sci. 1954;57(6):722-733.

91. Gomez F, Galvan RR, Cravioto J, Frenk S, Santaella JV, De La Pena C. Fat absorption in chronic severe malnutrition in children. Lancet. 1956;271(6934):121-122.

92. Robinson U, Behar M, Viteri F, Arroyave G, Scrimshaw NS. Protein and fat balance studies in children recovering from kwashiorkor. J Trop Pediatr (Lond). 1957;2(4):217-223.
93. Teotia M, Teotia SP, Sharma NL. Fat balance studies in marasmus. Indian JMed Res. 1969;57(12):2240-2245.

94. Amin K, Walia BN, Ghai OP. Small bowel functions and structure in malnourished children. Indian Pediatr. 1969;6(2):67-72.

95. Viteri FE, Flores JM, Alvarado J, Behar M. Intestinal malabsorption in malnourished children before and during recovery. Relation between severity of protein deficiency and the malabsorption process. Am J Dig Dis. 1973;18(3):201-211.

96. Reddy V. Fat-soluble vitamin deficiencies in children in relation to protein energy malnutrition and environmental stress. Prog Clin Biol Res. 1981;77:109-117.

97. Dammin GJ. The pathogenesis of acute diarrhoeal disease in early life. Bull World Health Organ. 1964;31:29-32.

98. Mata LJ, et al. Gastrointestinal flora of children with protein - calorie malnutrition. Am J Clin Nutr. 1972;25(10):118-126.

99. Gracey M, Stone DE. Small-intestinal microflora in Autralian Aboriginal children with chronic diarrhoea. Aust N Z J Med.1972;2(3):215-219.

100.Gracey M, Suharjono, Sunoto, Stone DE. Microbial contamination of the gut: another feature of malnutrition. Am JClin Nutr. 1973;26(11):1170-1174.

101. Maffei HV, Nóbrega FJ. Gastric pH and microflora of normal and diarrhoeic infants. Gut. 1975;16(9):719-726.

102. Heyworth B, Brown J. Jejunal microflora in malnourished Gambian children. Arch Dis Child. 1975;50(1):27-33

103. Ashworth A. Efficacy and effectiveness of community-based treatment of severe malnutrition. Food Nutr Bull. 2006;27(3 Suppl):S24-S48.

104.Schoonees A, Lombard M, Musekiwa A, Nel E, Volmink J. Ready-to-use therapeutic food for home-based treatment of severe acute malnutrition in children from six months to five years of age. Cochrane Database Syst Rev. 2013;(6):CD009000.

105. Lenters LM, Wazny K, Webb P, Ahmed T, Bhutta ZA. Treatment of severe and moderate acute malnutrition in low- and middle-income settings: a systematic review, meta-analysis and Delphi process. BMC Public Health. 2013;13(suppl 3):S23.

106.Zhang $\mathrm{L}$, et al. Impaired bile acid homeostasis in children with severe acute malnutrition. PLoS One. 2016;11(5):e0155143.

107. Yang L, et al. Long-term calorie restriction enhances cellular quality-control processes in human skeletal muscle. Cell Rep. 2016;14(3):422-428.

108. Preidis GA, Keaton MA, Campeau PM, Bessard BC, Conner ME, Hotez PJ. The undernourished neonatal mouse metabolome reveals evidence of liver and biliary dysfunction, inflammation, and oxidative stress. J Nutr. 2014;144(3):273-281.

109. Brown EM, et al. Diet and specific microbial exposure trigger features of environmental enteropathy in a novel murine model. Nat Commun. 2015;6:7806.

110. van Zutphen T, et al. Malnutrition-associated liver steatosis and ATP depletion is caused by peroxisomal and mitochondrial dysfunction. J Hepatol. 2016;65(6):1198-1208.

111. Jackevicius CA, Tu JV, Ross JS, Ko DT, Carreon D, Krumholz HM. Use of fibrates in the United States 
and Canada. JAMA. 2011;305(12):1217-1224.

112. ACCORD Study Group: Ginsberg HN, et al. Effects of combination lipid therapy in type 2 diabetes mellitus. NEngl JMed. 2010;362(17):1563-1574.

113. Markham A, Keam SJ. Obeticholic acid: first global approval. Drugs. 2016;76(12):1221-1226.

114. McGarry JD. What if Minkowski had been ageusic? An alternative angle on diabetes. Science. 1992;258(5083):766-770.

115. Moore DD. Nuclear receptors reverse McGarry's vicious cycle to insulin resistance. Cell Metab. 2012;15(5):615-622.

116. Haluzik MM, et al. Improvement of insulin sensitivity after peroxisome proliferator-activated receptor- $\alpha$ agonist treatment is accompanied by paradoxical increase of circulating resistin levels. Endocrinology. 2006;147(9):4517-4524.

117. Damci T, Tatliagac S, Osar Z, Ilkova H. Fenofibrate treatment is associated with better glycemic control and lower serum leptin and insulin levels in type 2 diabetic patients with hypertriglyceridemia. Eur JIntern Med. 2003;14(6):357-360.

118. Wan Q, et al. Regression to normoglycaemia by fenofibrate in pre-diabetic subjects complicated with hypertriglyceridaemia: a prospective randomized controlled trial. Diabet Med. 2010;27(11):1312-1317.

119. Li XM, Li Y, Zhang NN, Xie YH, Shi YQ. Combination therapy with metformin and fenofibrate for insulin resistance in obesity. JInt Med Res. 2011;39(5):1876-1882.

120. Buldak L, Dulawa-Buldak A, Labuzek K, Okopien B. Effects of 90-day hypolipidemic treatment on insulin resistance, adipokines and proinflammatory cytokines in patients with mixed hyperlipidemia and impaired fasting glucose. Int J Clin Pharmacol Ther. 2012;50(11):805-813.

121. Fabbrini E, et al. Effect of fenofibrate and niacin on intrahepatic triglyceride content, very low-density lipoprotein kinetics, and insulin action in obese subjects with nonalcoholic fatty liver disease. J Clin Endocrinol Metab. 2010;95(6):2727-2735.

122.Abbasi F, Chen YD, Farin HM, Lamendola C, Reaven GM. Comparison of three treatment approaches to decreasing cardiovascular disease risk in nondiabetic insulin-resistant dyslipidemic subjects. Am J Cardiol. 2008;102(1):64-69.

123. Belfort R, Berria R, Cornell J, Cusi K. Fenofibrate reduces systemic inflammation markers independent of its effects on lipid and glucose metabolism in patients with the metabolic syndrome. JClin Endocrinol Metab. 2010;95(2):829-836.

124. Black RN, Ennis CN, Young IS, Hunter SJ, Atkinson AB, Bell PM. The peroxisome proliferatoractivated receptor alpha agonist fenofibrate has no effect on insulin sensitivity compared to atorvastatin in type 2 diabetes mellitus; a randomised, double-blind controlled trial. J Diabetes Complicat. 2014;28(3):323-327. 